\title{
Mechanical Vibration Mechanism Motion Simulation of Ultrasonic Composite Vibration Polishing Equipment
}

\author{
Wan Hongqiang, ${ }^{\text {a }}$, Han Peiying, Ge Shuai, Li Fancong and Zhang Simiao \\ School of Mechanical Engineering, Xi'an Technology University, Xi'an 710021, China
}

\begin{abstract}
In order to design a kind of mechanical vibration part with stable motion in ultrasonic composite vibration polishing equipment. firstly, the main working principle of ultrasonic composite vibration polishing equipment is briefly introduced. Secondly, the mechanical vibration structure of the design is analyzed theoretically, again, using ADAMS software to simulate the crankshaft connecting rod piston mechanism. When the crankshaft speed is $750 \mathrm{r} / \mathrm{min}$, the stroke of the piston is $90 \mathrm{~mm}$, which is similar to the theoretical analysis value. Finally, using ANSYS Workbench software to analyze the crankshaft statics. The analysis shows that the design of the mechanical vibration structure is reasonable, which provides a theoretical basis for the study of the mechanical vibration structure of ultrasonic composite vibration polishing equipment.
\end{abstract}

\section{Introduction}

With the development of other disciplines such as aerospace, VLSI and optical instruments, the quality requirements for the surface of the key parts needing precision machining are more and more high, and the characteristic dimensions of the substrates used in the IC circuits have reached the $45 \mathrm{~nm}[1]$ with the large scale development trend. Traditional parts polishing relies on the experience of the workers to complete, but its processing cycle is long and low efficiency, and its processing quality is not stable [2], the current mainstream polishing method is composite vibration polishing, mainly including ultrasonic electrochemical composite polishing, ultrasonic-chemical composite polishing, ultrasonic auxiliary mechanical vibration composite polishing, ultrasonic fluid jet composite polishing, ultrasonic magnetic abrasive composite polishing and other polishing methods [3-7], but these polishing methods for the current requirements still have deficiencies. So domestic and foreign research institutions are still actively exploring new methods which have high-quality, high efficiency and low-cost surface processing.

In this paper, a composite surface machining technique combining ultrasonic machining, vibration processing and free abrasive machining is presented. Because the stability of mechanical vibration structure of ultrasonic compound vibration polishing equipment directly affects the polishing quality of workpiece surface, the mechanical vibration part of ultrasonic compound vibration polishing equipment is analyzed in this paper.

\footnotetext{
${ }^{a}$ Corresponding author : 413162931@qq.com; Tel:08613363941134
} 


\section{Working principle of ultrasonic composite vibrating polishing equipment}

Ultrasonic composite Vibration polishing working principle as shown in Figure 1., the workpiece is driven up and down by the mechanical vibration mechanism, and the workpiece is polished by the high speed relative motion of the abrasive and workpiece, and the energy assisted by the cavitation effect of ultrasonic vibration is used to remove the workpiece surface material by the aid of ultrasonic vibration, to achieve the workpiece surface polishing purposes.

\section{Structural design of mechanical vibration part}

The mechanical vibration part of ultrasonic compound vibrating polishing equipment is mainly connected with crankshaft connecting rod by crankshaft, connecting the piston through crank connecting rod, connecting clamp under the piston, and with the driving of crankshaft, the fixture is vibrating up and down in vertical direction. The mechanical vibration mechanism is the evolution of the crank-connecting rod structure, so the crank-piston mechanism can be simplified to crank-slider mechanism for theoretical analysis, and its structure diagram is shown in Figure 2.

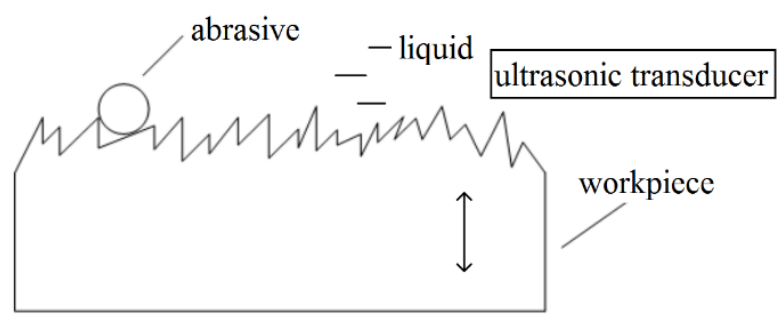

Figure 1. Schematic diagram of ultrasonic composite polishing process.

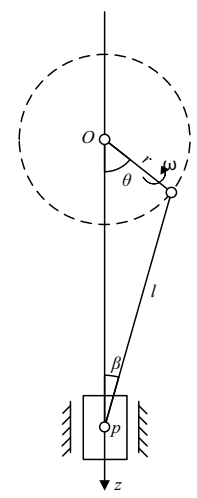

Figure 2. Mechanism sketch of crank slider.

Wherein, " $r$ " is the crank length, the " $l$ " is the connecting rod length, the point $\mathrm{P}$ is the fixture particle, " $O$ " is the crank rotation center, and " $\theta$ " is the angle that the crank rotates from the initial position, the " $\beta$ " is the angle that the connecting rod rotates from the initial position, and " $z$ " is the displacement of the jig. In this paper, the relation between the displacement, velocity, acceleration and $\theta$ of the slider is mainly studied.

The displacement expression (1) of the slider is based on the triangular relation, which is based on the 2 schematic diagram of the crank-slider structure, namely.

$$
\begin{aligned}
& z=r \cos \theta+\sqrt{l^{2}-r^{2} \sin ^{2} \theta} \\
& \quad \theta=\omega t \\
& \omega=\frac{2 \pi}{T}=2 \pi n
\end{aligned}
$$

In the formula, $\omega$--the angular velocity of the crank,

$t$--rotation time

$T$--rotation cycle,

$n$--the speed of the driving motor of mechanical vibrating mechanism.

The speed of the slider can be expressed by (4).

$$
v=\frac{d z}{d t}=\frac{d z}{d \theta} \cdot \frac{d \theta}{d t}=\omega \cdot \frac{d z}{d \theta}
$$


In the type, " $v "$ is the speed of the slider.

Substituting (1), (2) into (4), we can get the slider-type velocity expression.

$$
v=-\omega r \cdot \sin \theta \cdot\left[1+\frac{r \cos \theta}{\sqrt{l^{2}-r^{2} \sin ^{2} \theta}}\right]
$$

Because the acceleration of the slider can be expressed by the following expression.

$$
a=\frac{d v}{d t}=\frac{d v}{d \theta} \cdot \frac{d \theta}{d t}=\omega \frac{d v}{d \theta}
$$

In the formula, " $a$ " is the acceleration of the slider.

Therefore, substituting (5) into (6) can be used to get the acceleration expression of the slider (7).

$$
a=-\omega^{2} r\left[\cos \theta+\frac{r\left(l^{2} \cos 2 \theta+\mathrm{r}^{2} \sin ^{4} \theta\right)}{\left(l^{2}-\mathrm{r}^{2} \sin ^{2} \theta\right)^{3 / 2}}\right]
$$

The rotating speed of mechanical vibrating mechanism is $750 \mathrm{r} / \mathrm{min}$, the length of crank is $r=45 \mathrm{~mm}$, the length of connecting rod is $l=150 \mathrm{~mm}$. When the clamp reaches the maximum displacement, the maximum displacement is $z=195 \mathrm{~mm}$, the corresponding velocity and the acceleration value are $v=0 \mathrm{~m} / \mathrm{s}, \quad a=3.6 \times 10^{5} \mathrm{~mm} / \mathrm{s}^{2}$ respectively, when the rotation angle, the jig achieves the smallest displacement, its minimum displacement $z=105 \mathrm{~mm}$, the corresponding velocity and the acceleration value respectively are $v=0 \mathrm{~m} / \mathrm{s}, a=-1.97 \times 10^{5} \mathrm{~mm} / \mathrm{s}^{2}$. The stroke of the slider is $90 \mathrm{~mm}$.

\section{Kinematic Simulation and dimension optimization of crank mechanism}

In the ultrasonic compound vibrating polishing equipment, the mechanical vibration part plays a vital role, the polishing of workpiece is the result of the interaction between the mechanical vibration part and the ultrasonic vibration part, so it is necessary to carry on the motion simulation to the mechanical vibration part. For the convenience of analysis, this paper simulates the mechanical vibration part by using the software of Adams. The model of crankshaft linkage piston mechanism is established in Soildworks software, which is saved as Parasoild format and imported into Adams software, and the model of crankshaft linkage piston mechanism after import is shown in Figure 3.

The model of the crankshaft connecting rod piston is applied to constrain and drive. The model unit is set to MMKS units, and the gravity is negative in the $\mathrm{Y}$ axis, with a value of 9.806 .

\subsection{Add constraint}

-The title is set in bold 14-point Arial, flush left, unjustified. The first letter of the title should be capitalised with the rest in lower case. You should leave $35 \mathrm{~mm}$ of space above the title and $6 \mathrm{~mm}$ after the title.

-Add a rotation pair. The rotation pair between the ground and the crankshaft, the rotating pair between the connecting rod and the crankshaft, the rotating pair between the connecting rod and the piston pin are added respectively.

-Adds a mobile pair. Add a moving pair between the ground and the piston.

-Add a fixed constraint. Adding a fixed pair between the connecting rod cover and the bolt, a fixed pair between the connecting rod lid and the connecting rod, a fixed pair between the piston pin and the piston.

-Adds a mobile pair. Add the piston relative to the ground of the moving pair.

\subsection{Add Driver}

Add a constraint at one end of the crankshaft and the rotational speed is set to $750 \mathrm{r} / \mathrm{min}$. 
Add constraints and driven models as shown in Figure 4.

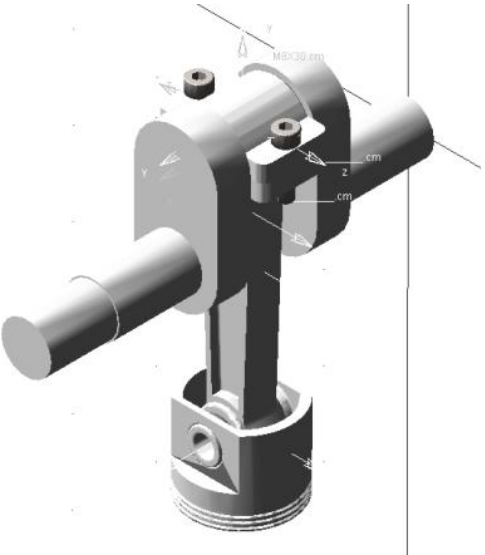

Figure 3. The model of the piston mechanism of the crankshaft connecting rod in ADAMS.
Figure 4. Impose constraints and drives on the model.

The displacement, velocity and acceleration of the piston are simulated. The simulation time is two cycles $0.16 \mathrm{~s}$, and in order to make the simulation data more precise, the simulation steps are 100 steps. The simulation results are shown in Figure 5 -7. respectively.

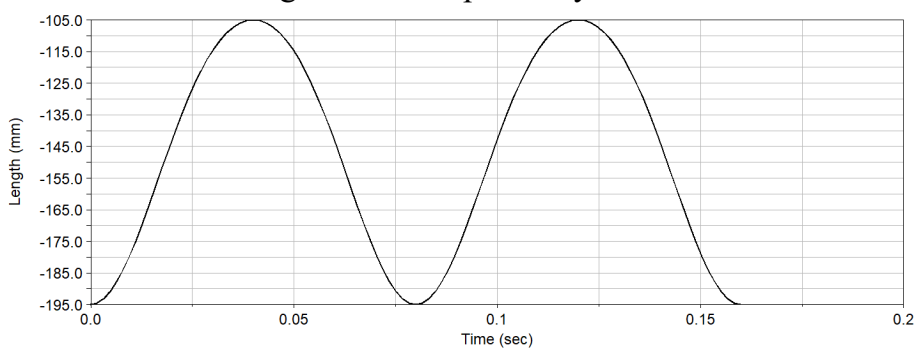

Figure 5. Displacement diagram of the piston.

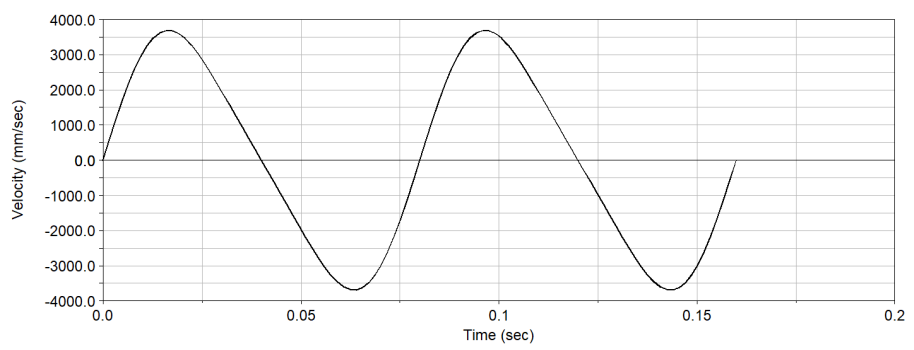

Figure 6. Velocity diagram of the piston.

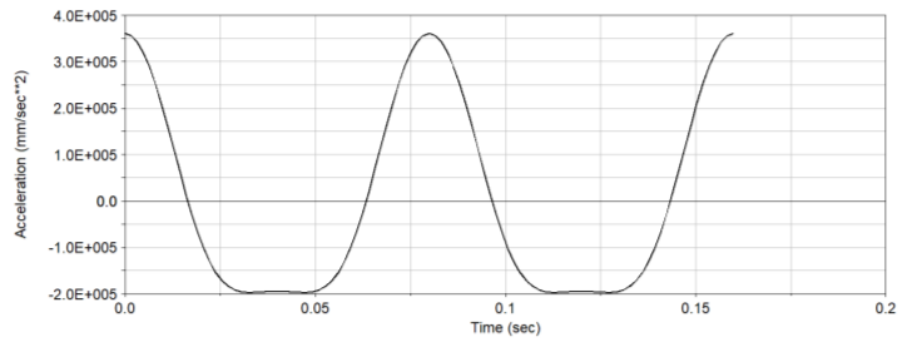

Figure 7. Acceleration diagram of the piston. 
As can be seen from Figure 9, the displacement and velocity curves of the piston are sinusoidal distributions, and the change interval of displacement is $[-195,-105] \mathrm{mm}$, and the change interval of velocity is $[-3686.4,3686.4] \mathrm{mm} / \mathrm{s}$. Therefore, we can calculate the piston stroke of $90 \mathrm{~mm}$, which is consistent with the theoretical calculation value, which shows that the above theoretical analysis is correct. The acceleration change interval of the piston is $\left[-1.97 \times 10^{5}, 3.6 \times 10^{5}\right] \mathrm{mm} / \mathrm{s}^{2}$. The displacement, velocity and acceleration of piston are stable with time curve in y direction and there is no obvious fluctuation. The simulation result is reasonable and the expected result is achieved.

\section{Finite element analysis of crankshaft}

In order to study whether the strength of crankshaft is up to the requirement, Ansys Workbench is used to analyze the crankshaft stress. The following are the specific implementation processes.

-Import model. After saving the crankshaft model drawn in Soildworks as Parasoild format, the static analysis is introduced into the Ansys Workbench.

-Set the crankshaft parameters. The crankshaft material chooses the ductile iron, and its elastic modulus is $173 \mathrm{GPa}$, Poisson's ratio is 0.3 , the density is $7.3 \mathrm{~g} / \mathrm{cm}^{3}$, the connecting rod material chooses the alloy steel, its elastic modulus is $206 \mathrm{GPa}$, the Poisson's ratio is 0.3 , the density is $7.9 \mathrm{~g} / \mathrm{cm}^{3}$, the bolt and the piston pin material chooses the carbon steel, and its elastic modulus is 206GPa, Poisson's ratio is 0.24 , density is $7.3 \mathrm{~g} / \mathrm{cm}^{3}$, piston material chooses cast iron, its elastic modulus is $126 \mathrm{GPa}$, Poisson's ratio is 0.24 , density is $7.0 \mathrm{~g} / \mathrm{cm}^{3}$

-Dividing the grid. By using the Hexahedral grid method, the cell meshes are divided into 5mm, the number of nodes is 54, 476, and the number of units is 28,760 , as shown in Figure 8.

-Imposing constraints and loads. The left end face of the crankshaft is fully restrained, the housing is formed on the left side of the crankshaft, the drive is added to the right side of the crankshaft, the torque is $15529 \mathrm{~N} \cdot \mathrm{mm}$, and the acceleration of gravity is added. As shown in Figure 9.

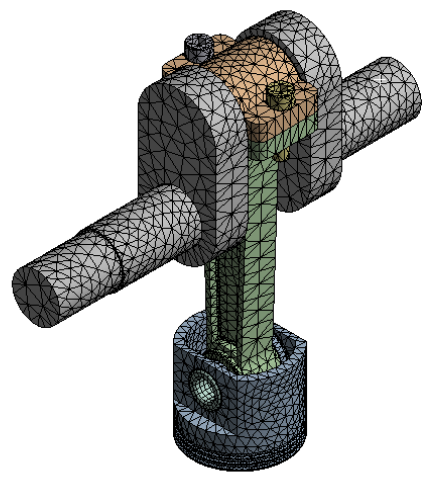

Figure 8. Meshing of the model of the crankshaft, connecting-rod and piston.

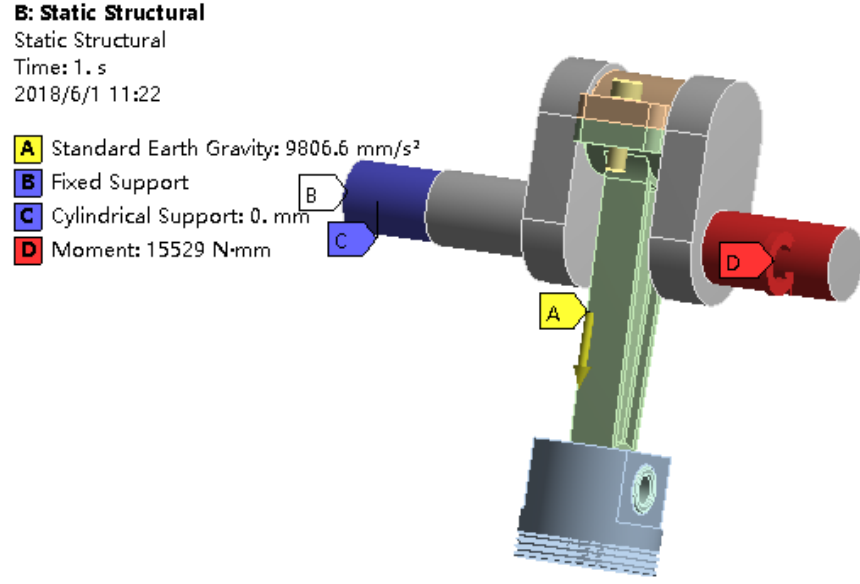

Figure 9. Applys the restraint and load.

-Solving and post processing. The total deformation analysis and the stress cloud of the crankshaft connecting rod piston mechanism are obtained by using the Solve command. As shown in figures 10 . and figure 11.

As can be seen from the total displacement diagram, the maximum displacement deformation of the crankshaft is the largest, and the maximum displacement deformation is $9.12 \times 10^{-3} \mathrm{~mm}$, from the equal effect, it can be seen that the stress and deformation at the contact boundary between the crankshaft shaft neck, the bolt and the connecting rod, the piston pin and the connecting rod and the piston are the largest $27.307 \mathrm{MPa}$. Because the material of the crankshaft is carbon-graphite cast iron, its allowable stress value is $320 \mathrm{MPa}$ and $320 \mathrm{MPa}>27.307 \mathrm{MPa}$, so the crankshaft connecting rod piston mechanism satisfies the design requirement. 


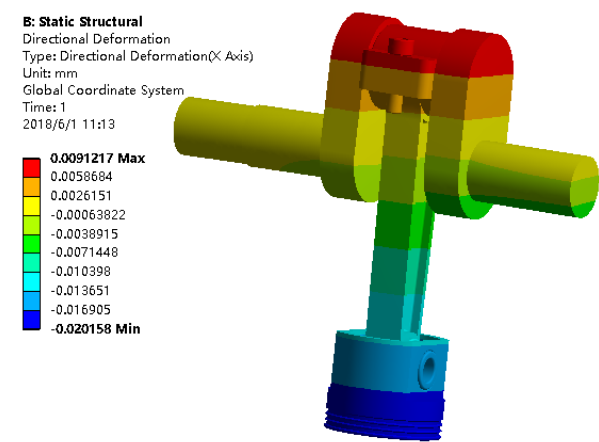

Figure 10. Total deformation.

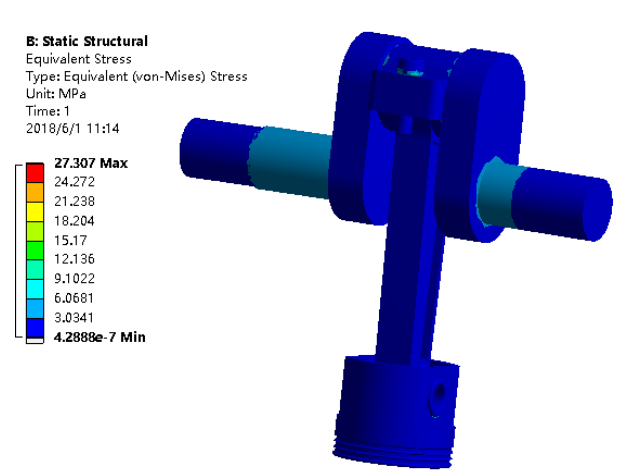

Figure 11. Equivalent stress.

\section{Conclusions}

The theoretical analysis and calculation of the crank-rod piston mechanism model is provided to provide theoretical basis for the design of ultrasonic composite vibration polishing equipment. The model of crankshaft linkage piston mechanism established in Soildworks software is introduced into Adams software, and the velocity, acceleration and displacement curves of piston are analyzed. The results are similar to the theoretical calculation, when the crankshaft speed is $750 \mathrm{r} / \mathrm{min}$, the stroke of the piston is $90 \mathrm{~mm}$. In order to study whether the crankshaft strength satisfies the requirement, the stress analysis of the crankshaft connecting rod piston mechanism is carried out by using the Ansys Workbench software, the maximum stress and deformation of crankshaft is $27.307 \mathrm{MPa}$, which is less than the allowable stress of crankshaft $320 \mathrm{MPa}$, so the strength of crankshaft satisfies the requirement. This study provides a new design idea and theoretical basis for similar ultrasonic composite vibrating polishing mechanism.

\section{Acknowledgement}

This study is funded by Scientific research project of Key Laboratory of Shaanxi Provincial Department of Education (16JS047).

This study is funded by Science and Technology Research and Development Program of Shaanxi Province(2016GY-013).

\section{References}

1. Li Liming, Li Mao, Zhu Yongwei. Research status and prospect of consolidation abrasive grinding and polishing [J]. Diamond and Abrasives Engineering, 2009, (05): 17-22.

2. Chen Yu Jun, Tangyu, Miao want to light, Yin Hui. A review of abrasive jet surface polishing [J]. Surface Technology, 2015, 44 (11): 119-127.

3. GUO Z N, LEE T C, YUE T M, et al. The Design of an Ultrasonic Polishing Tool by the Transfer-matrix Method [J]. Materials Processing Technology, 2000, (102): 122-127.

4. SINGH R, KHAMBA J S. Ultrasonic Machining of Titanium and Its Alloys: A Review [J]. Materials Processing Technology, 2006(6): 125-135.

5. ZHAN J M, ZHAO J. Study of the Contact Force in Free-form-surfaces Compliant EDM Polishing by Robot [J]. Materials Processing Technology, 2002(129): 186-189.

6. Sun Qi. Experimental study on ultrasonic-electrochemical polishing of silicon carbide by experiment [D].Harbin: Harbin Institute of Technology, 2016.

7. Li Ren Chuang. Experimental study on grinding and polishing of jade surface by ultrasonic Vibration auxiliary device [D].Liaoning: Liaoning University of Science and Technology, 2017. 